05,13

\title{
Модовая фильтрация поверхностных магнитостатических волн в YIG/FeRh
}

\author{
(C) С.А. Одинцов ${ }^{1}$, А.А. Амиров ${ }^{2,3}$, А.А. Грачев ${ }^{1}$, В.В. Родионова ${ }^{2}$, А.В. Садовников ${ }^{1}$ \\ ${ }^{1}$ Саратовский государственный университет им. Н.Г. Чернышевского, \\ Саратов, Россия \\ ${ }^{2}$ Балтийский федеральный университет им. И. Канта, \\ Калининград, Россия \\ ${ }^{3}$ Институт фризики Дагестанского федерального исследовательского центра РАН, \\ Махачкала, Россия \\ E-mail: odinoff@gmail.com
}

Поступила в Редакцию 9 апреля 2021 г.

В окончательной редакции 9 апреля 2021 г.

Принята к публикации 19 апреля 2021 г.

\begin{abstract}
Проведено численное исследование особенностей распространения спиновых волн в волноводе из железоиттриевого граната (ЖИГ) и сплава $\mathrm{Fe}-\mathrm{Rh}$ в форме пластины, расположенного поверх центральной части ЖИГ. На основе результатов моделирования также выявлены возможности управления динамикой спиновых волн в исследуемой структуре. Микромагнитное численное моделирование использовалось для исследования перекачки спин-волнового сигнала в многомодовом режиме путем численного решения уравнения Ландау-Лифшица-Гильберта. Преобразование спектров пропускания спиновых волн показывает, что предложенная структура позволит управлять распространением спин-волновых мод за счет резкого изменения намагниченности $\mathrm{Fe}-\mathrm{Rh}$ в области температуры магнитного фазового перехода, близкого к комнатной температуре. Кроме того, спин-волновой сигнал может управляться посредством небольшого изменения температуры в пластине $\mathrm{Fe}-\mathrm{Rh}$, создаваемого посредством лазерного излучения.

Двухслойная структура ЖИГ/Fe-Rh, с прикладной точки зрения, может быть использована в качестве функциональной единицы в планарных магнонных сетях, выполняющих пространственно-частотное демультиплексирование и режим фильтрации спин-волновых мод.
\end{abstract}

Ключевые слова: спиновые волны, магноника, нагрев.

DOI: 10.21883/FTT.2021.09.51307.24H

\section{1. Введение}

В последнее время активно изучаются магнитные материалы с магнитным фазовым переходом первого рода (FОМРТ) в связи с перспективами применения их в энергоэффективных технологиях, системах контроля температуры, устройствах памяти и спинтронике $[1,2]$. Интерметаллический сплав $\mathrm{Fe}-\mathrm{Rh}$ является одним из перспективных материалов с гигантским магнитокалорическим эффектом (MCE) [3] и колоссальным магнитосопротивлением (CMR) при температуре выше комнатной [4]. Как известно, сплавы Fe-Rh с составами близкими к эквиатомным, характеризуются упорядоченной кристаллической структурой типа $\mathrm{CsCl}$, которая характеризуется изменением намагниченности [5] параметров решетки [6] и теплоемкости [7] в диапазоне температур 310-360 К. Одна из важных проблем исследования материалов с FОМРТ связана с исследованием методики и механизмов управления свойствами магнетизма в них, и существует несколько способов изменения намагниченности и температуры FOMPT, такие как магнитное поле [8], гидростатическое давление [9], электрическое поле, индуцированное деформацией $[10,11]$ и другие. В свою очередь магнонный волновод, который сформирован из магнитной пленки в форме полосы, является строительным блоком любой сложной интегральной магнонной сети [12]. Подобные структуры уже зарекомендовали себя как линии передачи между устройствами обработки сигналов $[13,14]$.

Таким образом, комбинируя эти структуры, возможно добиться большего контроля над дисперсией и динамикой спиновых волн. В настоящей работе мы сообщаем об управлении спиновыми волнами, распространяющимися как направленные моды комбинированной структуры $\mathrm{YIG} / \mathrm{Fe}-\mathrm{Rh}$. Полоса Fe-Rh размещена на полосе ЖИГ и воздействует на внутреннее поле волновода ЖИГ. В результате микромагнитного численного моделирования были получены спектры и дисперсионные характеристики спиновых волн через магнонную структуру. Анализ полученных данных показал, что эту структуру можно использовать как функциональную единицу в планарных магнонных сетях, например, как модовый фильтр. Также показана возможность управления распространением мод спин-волнового сигнала благодаря изменению температуры слоя Fe-Rh.

\section{2. Исследуемая структура}

На рис. 1,a показано схематическое изображение исследуемой структуры, которая представляет из 

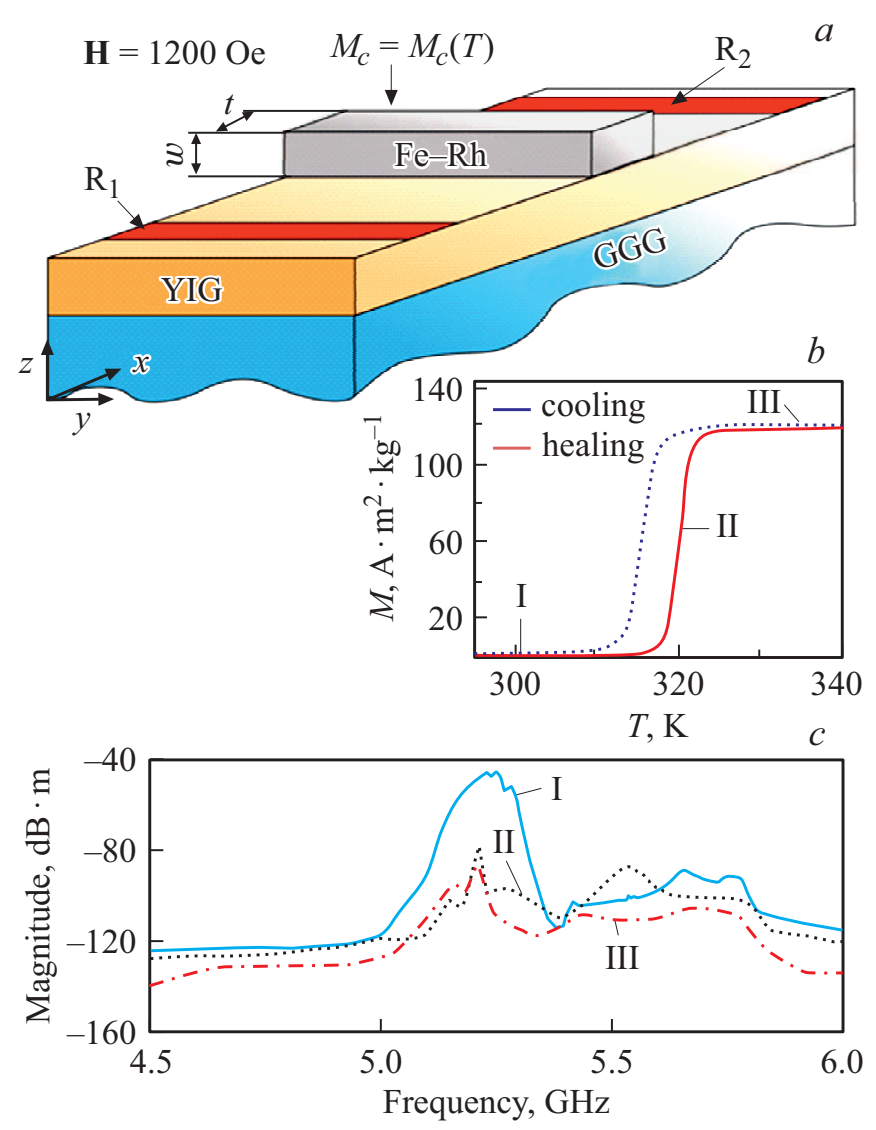

Pис. 1. $a-$ схема рассматриваемой структуры; $b-$ экспериментально рассчитанная температурная зависимость намагниченности сплава $\mathrm{Fe}_{48} \mathrm{Rh}_{52}$. (красная линия - нагрев, синяя линяя - охлаждение); $c$ - частотная зависимость амплитуды спиновой волны при разных значениях намагниченности сплава $\mathrm{Fe}_{48} \mathrm{Rh}_{52}$ в исследуемой структуре.

себя микроволновод выполненный из ЖИГ [(YIG) $\left.\mathrm{Y}_{3} \mathrm{Fe}_{5} \mathrm{O}_{12}(111)\right]$, выращенный на подложке из галлийгадолиниевого граната $\left[(\mathrm{GGG}) \mathrm{Gd}_{3} \mathrm{Ga}_{5} \mathrm{O}_{12}\right.$ (111)]. Толщина ЖИГ микроволновода принимается за $10 \mathrm{mkm}$, ширина $1 \mathrm{~mm}$, Длина всей структуры составляет $10 \mathrm{~mm}$. На ЖИГ волноводе, во всю ширину, расположена полоса из сплава Fe-Rh, таким образом, что он лежит ровно посередине структуры вдоль оси $x$. Также на рис. $1, a$ областью $R_{1}$ показана область возбуждения спиновых волн (CВ), а областью $R_{2}$ показана область снятия сигнала при численном моделировании. Структура помещена во внешнее статическое магнитное поле, $H_{0}=1200 \mathrm{Oe}$, ориентированное вдоль оси у для эффективного возбуждения поверхностной магнитостатической волны (ПМСВ). В ходе численного моделирования размеры полосы $\mathrm{Fe}-\mathrm{Rh}$ изменялись, для выявлений эффектов воздействия геометрических масштабов Fe-Rh на распространение спиновых волн в ЖИГ микроволноводе. Для сплавов состава Fe-Rh с составом близким к эквиатомным и упорядоченного в структуру типа $\mathrm{CsCl}$, характерен изоструктурный метамагнитный переход из антиферромагнитной (АФМ) фазы в ферромагнитную (ФМ) фазу при температурах близких к комнатной, вследствие чего наблюдается резкое изменение намагниченности [15]. Для данного исследования были использованы характеристики сплава $\mathrm{Fe}_{48} \mathrm{Rh}_{52}$ [10], зависимость намагниченности от температуры которого показана на рис. 1, $b$. Для численного моделирования были выбраны три характерных точки, отмеченные на рис. $1, b$, которые соответствуют трем уровням температуры/намагниченности в Fe-Rh, а именно (I) $M_{\text {sat }}=0$, (II) $M_{\text {sat }}=M_{\max } / 2$ и (III) $M_{\text {sat }}=M_{\max }$.

\section{3. Численное моделирование}

Для исследования распространения спиновых волн в исследуемой структуре было проведено микромагнитное моделирование в программе MuMax3 [16] на основе численного решения уравнения Ландау-ЛифшицаГильберта методом Дормана-Принса, которое описывает прецессию магнитного момента $M$ в эффективном магнитном поле $H_{e f f}=H_{0}+H_{\text {demag }}+H_{e x}+H_{a}$, где $H_{0}-$ внешнее магнитное поле, $H_{\text {demag }}-$ поле размагничивания, $H_{e x}$ - обменное поле, $H_{a}$ - поле анизотропии. При этом поле анизотропии полагалось равным $H_{a}=0$, поскольку вектор равновесной намагниченности направлен вдоль осей симметрии ЖИГ. Для уменьшения отражений сигнала от границ расчетной области в численном моделировании были введены регионы с увеличивающимся в геометрической прогрессии коэффициентом затухания $\alpha=10^{-5}$ в начале входной и в конце выходной секций волноведущей структуры.

На рис. 1, $c$ представлены результаты микромагнитного моделирования в виде спектра прохождения спиновой волны в исследуемой структуре, при значениях намагниченности отмеченными на рис. $1, b$. В случае, когда намагниченность сплава Fe-Rh минимальна, его влияние на распространение также минимально, в связи с этим, спектр имеет достаточно широкую полосу прохождения спиновой волны на выходной области структуры. В отличии от случаев, когда происходит изменение намагниченности, в связи с нагревом железо-родия. Так, чем выше намагниченность, тем хуже прохождение сигнала, вплоть до полного затухания волны после блока $\mathrm{Fe}-\mathrm{Rh}$.

В ходе исследования физических процессов, определяющих характеристики управления спин-волновыми сигналами путем создания изменения намагниченности в сплаве Fe-Rh, была разработана численная модель на основе метода конечных элементов (МКЭ). На данном этапе численного моделирования, в области возбуждения $R_{1}$ задавался входной сигнал с плавно нарастающей в момент включения амплитудой $b_{0}=0.01 \mathrm{mТ}$ и частотой $f$ в виде: $b_{z}(t)=b_{0} \sin (2 \pi f \cdot t)$. Полученные распределения компоненты динамической намагниченности $m_{z}^{2}+m_{x}^{2}$ приведены на верхних панелях рис. 2, $\left.a-c\right)$ для различных значений намагниченности блока сплава $\mathrm{Fe}-\mathrm{Rh}$ на частоте $5.2 \mathrm{GHz}$. В соответствии с обозначениями, введенными на рис. $1, b$, были выбраны три значения намагниченности $\mathrm{Fe}-\mathrm{Rh}$, которые соответствуют 


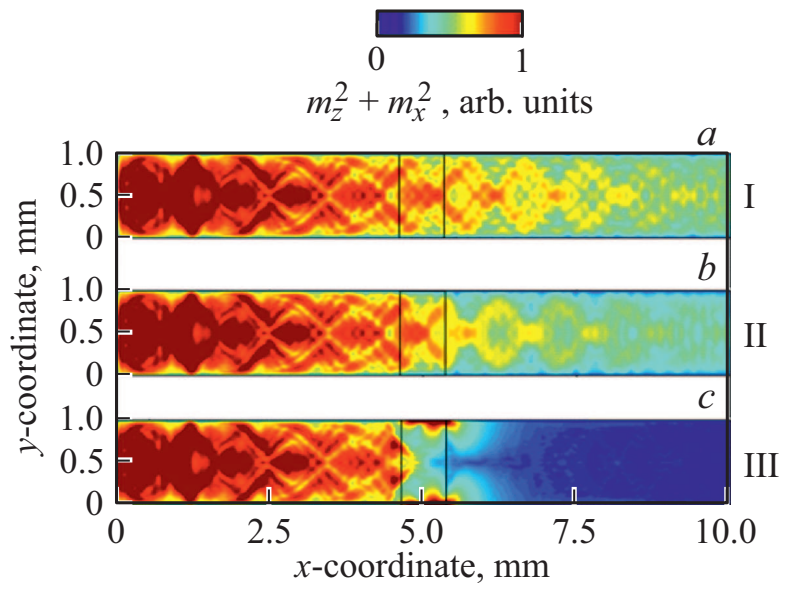

Рис. 2. Карты распределения динамической намагниченности при разных значениях намагниченности в блоке $\mathrm{Fe}_{48} \mathrm{Rh}_{52}$.

разным степеням нагрева материала. Таким образом, полученные карты намагниченности наглядно показывают процессы изменения динамики распространения спиновых волн в магнонной структуре, нагруженной блоком сплава Fe-Rh. Так, в случае I, т.е. при минимальном значении нагрева и намагниченности, распространению CB ничего не мешает. Однако при увеличении намагниченности до уровня, обозначенного на рис. 1, $c$ римской цифрой II, наблюдается изменение длины волны после прохождения блока $\mathrm{Fe}-\mathrm{Rh}$, что вызвано сильным влиянием последнего на распределение внутреннего магнитного поля Hint в исследуемой системе. И в случае максимальной намагниченности Fe-Rh (III) волна полностью затухает. Далее необходимо оценить влияние геометрических параметров блока железо-родия и его влияние на распределение внутреннего магнитного поля в системе, так как именно этот эффект является определяющим для управления эффектом модовой и частотной фильтрации в исследуемой системе. Для этого были произведены расчеты профилей внутреннего магнитного поля в исследуемой структуре для нескольких вариаций геометрических масштабов. На рис. 3, $а$ приведены распределения величины внутреннего магнитного поля $H_{\text {int }}$ в ЖИГ полоске (черная штриховая линия на рис. 3,a) вдоль оси $x$, в случае вариации геометрических параметров слоя $\mathrm{Fe}-\mathrm{Rh}$. Видно, что изменение толщины $t$ и ширины $w$ слоя $\mathrm{Fe}-\mathrm{Rh}$ производит значительное влияние на величину внутреннего магнитного поля $H_{\text {int }}$, в случае $t=200 \mu \mathrm{m}$ и $w=40 \mu \mathrm{m}$ (красная сплошная линия на рис. $3, a)$ происходит уменьшение величины $H_{\text {int }}$ в области слоя $\mathrm{Fe}-\mathrm{Rh}$ на величину 120 Ое. При увеличении ширины слоя Fe-Rh (синяя штрихпунктирная кривая на рис. $2, a) w=90 \mu \mathrm{m}$, в системе наблюдается более сильное влияние на величину внешнего магнитного поля в ЖИГ микроволноводе. Подобная трансформация величины $H_{\text {int }}$ изменяет дисперсию и характер распространения спиновых волн в ЖИГ полоске. С помощью
МКЭ был проведен расчет электродинамических характеристик композитной структуры. Были простроены дисперсионные характеристики первых мод спиновых волн для одиночного ЖИГ микроволновода (черная сплошная кривая на рис. $3, b)$ и композитной структуры ЖИГ/Fe-Rh (красная пунктирная кривая на рис. $3, b$ ). Взаимодействие слоя $\mathrm{Fe}-\mathrm{Rh}$ с ЖИГ микроволноводом приводит к изменению величины $H_{\text {int }}$ в структуре и происходит частотное смещение дисперсионных характеристик, распространяющихся в ней спиновых волн на величину $\Delta f$. Таким образом, нагрузка в виде блока $\mathrm{Fe}-\mathrm{Rh}$ расположенного над ЖИГ волноводом при нагревании создает провал в распределении внутреннего магнитного поля всей структуры. По этой причине дисперсионная характеристика спиновой волны которая распространяется вдоль такой структуры смещается вниз по частоте. В связи с этим длина волны также трансформируется вместе с ее модовым составом, что открывает перспективы не только к управлению этими волнами, но и к генерации коротких спиновых волн в такого рода структурах.

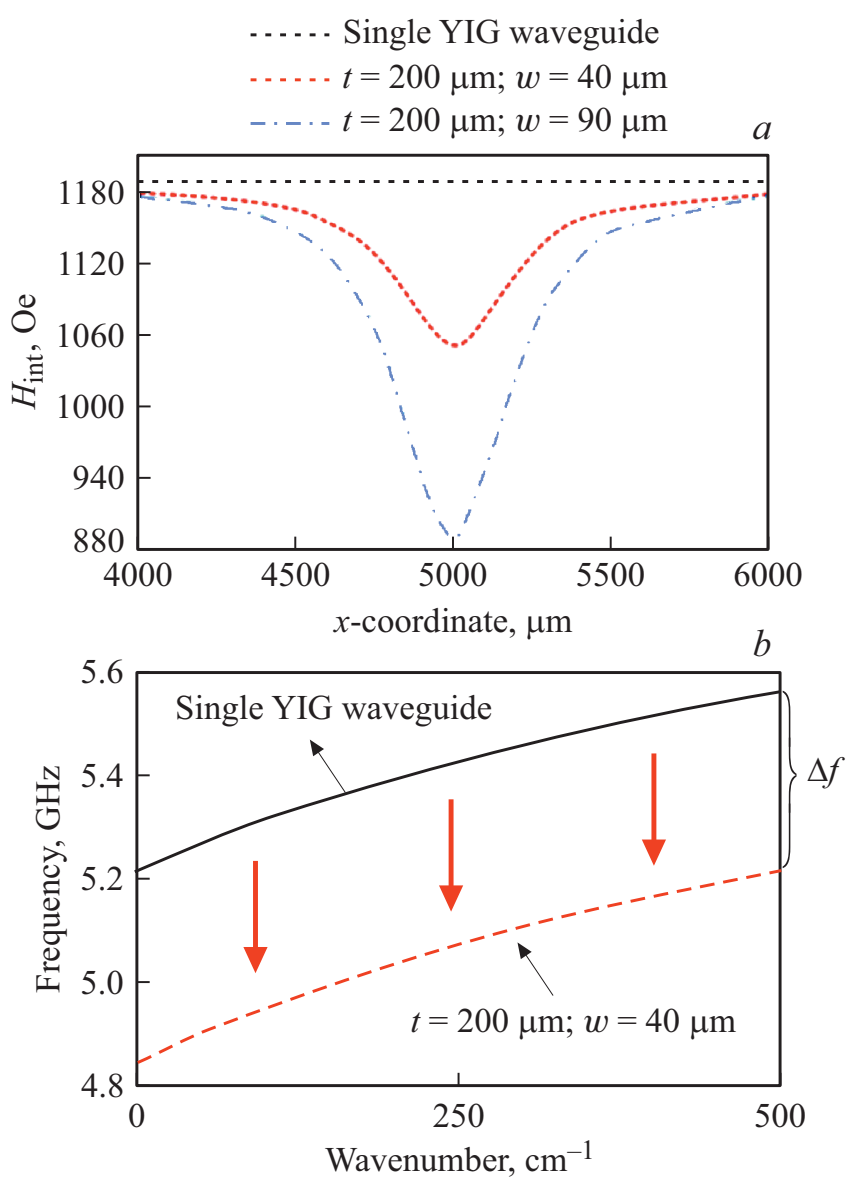

Рис. 3. $a-$ профили внутреннего магнитного поля $H)_{\mathrm{int}}$ в случаях вариации геометрических параметров сплава $\mathrm{Fe}_{48} \mathrm{Rh}_{52}$. Величина внешнего магнитного поля $H_{0}=1200 \mathrm{Oe} ; b-$ исперсионные характеристики при влиянии блока $\mathrm{Fe}_{48} \mathrm{Rh}_{52}$ и без него. 


\section{4. Заключение}

Таким образом, мы пронаблюдали модовую и частотную фильтрацию для управления спиновыми волнами, распространяющимися в полосе железо-иттриевого граната с полосой $\mathrm{Fe}-\mathrm{Rh}$ на ней. Микромагнитное численное моделирование выявило возможности управления переносом спиновых волн. Были подсчитаны спектры и дисперсионные характеристики спиновых волн в исследуемой магнонной структуре при разных значениях намагниченности геометрических параметров блока Fe-Rh. Рассчитаны характеристики фильтрации мод для поверхностной магнитостатической спиновой волны. Анализ результатов показал, что эта структура может использоваться как функциональная единица в планарных магнонных сетях. Из чего следует, что благодаря сильному влиянию Fe-Rh на внутреннее магнитное ЖИГ в такой структуре также возможно контролировать распространение спиновых волн моды, варьируя температуру слоя $\mathrm{Fe}-\mathrm{Rh}$.

\section{Финансирование работы}

Работа выполнена при поддержке Минобрнауки России в рамках выполнения государственного задания (проект № FSRR-2020-0005).

\section{Конфликт интересов}

Авторы заявляют, что у них нет конфликта интересов.

\section{Список литературы}

[1] J. Fullerton, J. Phys. D 50, 363001 (2017).

[2] V. Franco, J. Blasquez, J. Ipus, J. Law, L. Moreno-Ramirez, A. Conde. Prog. Mater. Sci. 93, 112 (2018).

[3] S. Nikitin, G. Myalikgulyev, A. Tishin, M. Annaorazov, K. Asatryan, A. Tyurin. Phys. Lett. A148, 363 (1990).

[4] Y. Lee, Z.Q. Liu, J.T. Heron, J.D. Clarkson, J. Hong, C. Ko, M.D. Biegalski, U. Aschauer, S.L. Hsu, M.E. Nowakowski, J. Wu, H.M. Christen, S. Salahuddin, J.B. Bokor, N.A. Spaldin, D.G. Schlom, R. Ramesh. Nature Commun. 6, 5959 (2015).

[5] A. Tohki, K. Aikoh, A. Iwase, K. Yoneda, S. Kosugi, K. Kume, T. Batchu-luun, R. Ishigami, T. Matsui. Appl. Phys. 111, 07A742 (2012).

[6] A.I. Zakharov, A.M. Kadomtsewa, R.Z. Levitin, E.G. Ponyatovskii. J. Exper. Theor. Phys. 19, 1348 (1964).

[7] M.P. Annaorazov, K.A. Asatryan, G. Myalikgulyev, S.A. Nikitin, A.M. Tishin, A.L. Tyurin. Cryogenics 32, 867 (1992).

[8] J.S. Kouvel. J. Appl. Phys. 37, 1257 (1966).

[9] R. Wayne. Phys. Rev. 170, 523 (1968).

[10] A.A. Amirov, V.V. Rodionov, I.A. Starkov, A.S. Starkov, A. Aliev. JMMM 470, 77-80 (2019).

[11] A.A. Amirov, I.A. Baraban, A.A. Grachev, A.P. Kamantsev, V.V. Rodionov, D.M. Yusupov, V.V. Rodionova, A.V. Sadovnikov. AIP Advances 10, 025124 (2020).

[12] V.V. Kruglyak, S.O. Demokritov, D. Grundler. J. Phys. D 43, 264001 (2010).
[13] A. Sadovnikov, E. Beginin, S. Odincov, S. Sheshukova, Y. Sharaevskii, A.I. Stognij, S. Nikitov. Appl. Phys. Lett. 108, 172411 (2016).

[14] A. Sadovnikov, A. Grachev, V. Gubanov, S. Odintsov, A. Martyshkin, S. Sheshukova, Y. Sharaevskii, S. Nikitov. Appl. Phys. Lett. 112, 142402 (2018).

[15] A.P. Kamantsev, V.V. Koledov, A.V. Mashirov, E.T. Dilmieva, V.G. Shavrov, J. Cwik, I.S. Tereshina, M.V. Lyange, V.V. Khovaylo, G. Porcari, M. Topic. Bull. Russ. Acad. Sci. Phys. 79, 1086 (2015).

[16] A. Vansteenkiste, J. Leliaert, M. Dvornik, M. Helsen, F. Garcia-Sanchez, B. Van Waeyenberge. AIP Advances 4, 107133 (2014).

Редактор К.В. Емцев 